\title{
Education for Radiological Technologists and Their Place in Mammography Diagnostic Methods
}

Tome Naumov ${ }^{\mathrm{a}}$

Elizabeta Popova Ramov

\author{
Snezana Stoilov
}

Leonid Ramov ${ }^{d}$

\begin{abstract}
Mammography is one of the screening methods used to detect breast cancer, which is established based on the protocols within a health system. However, many developing countries are unable to avail of this method. Although, other screening methods can be used in rural areas with portable equipment and well-trained radiology technologists. Our investigation aims to determinate the frequency of mammography in radiology departments as a basis for education of radiological technologists. Materials and methods: We performed a retrospective analysis of the frequency of mammography and mastectomies in the last three years at the Bitola clinical hospital in Macedonia. Results: Upon examining the procedures of 2,373 patients over the last three years, results indicate a high number of diagnosed and treated breast cancer patients. Radiological technologists are involved in the process of diagnosing and treating the disease and also can learn from other medical experts. Discussion: The radiation dose in modern mammography is not as high as in an MRI, and with precise protocols mammography can be used as a screening method in almost every hospital. Conclusion: University-educated radiological technologists can perform some diagnostic methods in rural areas without the supervision of a doctor. Education of these staff could have a huge economic benefit for society, particularly in rural areas in which, an easy access to a full-service hospital is not available.
\end{abstract}

Keywords: Breast Cancer • Mammography • Radiological Technologist

a Tome Naumov, Department of Physical Rehabilitation, University St. Clement Ohridski, Bul. Goce Delcev No.6, P.O. Box 566 Skopje 1000, Macedonia

Email: drnaumov@hotmail.com

b Corresponding author

Elizabeta Popova Ramova, Department of Physiotherapist Education, University St. Clement Ohridski, Bul. Goce Delcev No.6, P.O. Box 566 Skopje 1000, Macedonia

Research areas: Spine deformities; Osteoporosis; Muscles skeletal sonography; pain management; Light therapy

Email: betiramova62@yahoo.com

c Snezana Stoilova, Department of Physiotherapist Education, University St. Clement Ohridski, Bul. Goce Delcev No.6, P.O. Box 566 Skopje 1000, Macedonia Skopje 1000, Macedonia

d Leonid Ramov, Department of Physiotherapist Education, University Goce Delcev, Bul. Goce Delcev No.6, P.O. Box 566 Skopje 1000, Macedonia

Email: ramov@ugd.edu.mk 
There are about 1.38 million new cases and 458,000 deaths from breast cancer each year (IARC Globocan, 2008). Breast cancer is by far the most common cancer for the female population, in both developed and developing countries. In low- and middle-income countries, the number of new cases has been rising steadily due to increased life expectancy, urbanization, and adoption of Western lifestyles (National Cancer Institute of Canada, 2006).

Currently, there is insufficient knowledge regarding the causes of breast cancer; therefore, early detection of the disease remains the cornerstone of breast cancer control. If detected early and if adequate diagnosis and treatment are available, there is a good chance that the cancer can be eradicated. However, if detected late, curative treatment is often no longer an option. In these cases, palliative care to relieve the suffering of patients and their families is needed.

The majority of deaths $(269,000)$ occur in low- and middle-income countries, where most cases of breast cancer are detected and diagnosed in late stages due to a lack of awareness on early detection as well as barriers to health services (Coldman, Phillips, Warren, \& Kan, 2006).

The World Health Organization (WHO) promotes comprehensive breast cancer control programs as part of national cancer control plans. The recommended early detection strategies for low- and middle-income countries are awareness of early signs and symptoms and clinical screenings in demonstration areas. Traditional mammography screening is very costly and feasible only in countries with good health infrastructure that can afford a long-term program (Yaffe, 2004).

In resource-poor settings, the majority of women with breast cancer are diagnosed at advanced stages of the disease; their five-year survival rates are low, ranging from $10 \%$ to $40 \%$. In settings where early detection and basic treatment are available and accessible, the five-year survival rate for early localized breast cancer exceeds $80 \%$. Breast cancer can be detected early through two strategies: early diagnosis and screening. Early diagnosis is based on improved public and professional awareness of signs and symptoms associated with cancer; it entails recognizing possible warning signs of cancer and taking prompt action (WHO, 2014).

Screening involves the systematic use of testing, such as mammography, across an asymptomatic population to detect and treat cancer or pre-cancers. The 
educational program for radiological technologists in the High Medical School at University St. Clement Ohridski, special attention is given to mammography. Mammography for women under 30 years of age has an unfavorable benefit: risk ratio due to the challenges of detecting cancer in younger breasts, the aggressiveness of cancers at this age, the potential for radiation susceptibility at younger ages, and a greater cumulative radiation exposure. Mammography may confer greatly improved benefit: risk ratios, estimated to be about 220 to one, when used as follows: in combination with MRI for women who carry strong breast cancer susceptibility (e.g., BRCA1/2 carriers), and/or if begun at age 35 and continued for 35 years. While there is considerable uncertainty in the risk of radiation-induced breast cancer, the risk as it is expressed in published studies is conservative, as the radiation dose absorbed by women receiving modern mammography has been substantially reduced due to newer technology.

The aim of our investigation was to determine the frequency of mammography screenings in radiological departments, as a basis for education for radiological technologists, and to access the morbidity of the breast cancer surgery population that has been treated in the Bitola clinical hospital in Macedonia.

\section{Materials and Methods}

We collected data and retrospectively analyzed administrative documents and protocols of the Radiological and Surgery Department at the Bitola clinical hospital. This study should serve as a base for practical education for our students. We have investigated patients' mammography frequency by age and sex, whether or not they underwent mammography in last three years, and whether or not they had a mastectomy. We used statistical method $t$-test, with a significance of $p<.05$.

\section{Results}

Frequency of patients who underwent mammography grouped by sex is shown in Table 1. 
Table 1

Mammography Diagnostic Method in Last Three Years

\begin{tabular}{ccccccc}
\hline Year & Male & $\%$ & Female & $\%$ & Total & $\%$ \\
\hline 2011 & 43 & 5.7 & 711 & 94.3 & 754 & 32 \\
2012 & 66 & 8.7 & 688 & 91.3 & 754 & 32 \\
2013 & 41 & 4.7 & 824 & 95.3 & 865 & 36 \\
Total & 150 & 6.3 & 2223 & 97.3 & 2373 & 100 \\
\hline
\end{tabular}

Most patients were female (97.3\%), which is consistent with the worldwide demographic characteristic.

Table 2

Frequency of Surgery-Treated Patients after Mammography

\begin{tabular}{ccccccccc}
\hline Age & $N$ & $20-29$ & $30-34$ & $35-45$ & $45-54$ & $55-64$ & $65-74$ & $>75$ \\
\hline 2011 & 754 & 7 & 8 & 101 & 172 & 234 & 157 & 75 \\
2012 & 754 & 0 & 13 & 74 & 192 & 231 & 169 & 75 \\
2013 & 865 & 0 & 2 & 49 & 172 & 309 & 190 & 143 \\
Total & 2373 & 7 & 23 & 224 & 536 & 774 & 516 & 293 \\
$\%$ & 100 & 0.3 & 0.9 & 9.4 & 23 & 33 & 22 & 12 \\
\hline
\end{tabular}

Most patients treated with a mastectomy (33\%) were between 55 and 64 years of age, and $66 \%$ were in working health before treatment.

\section{Discussion}

Breast cancer is by far the most common cancer for the female population, in both developed and developing countries. In low- and middle-income countries, the number of new cases has been rising steadily due to increased life expectancy, increased urbanization, and adoption of Western lifestyles (National Cancer Institute of Canada, 2006). In 2000, the mortality rate of breast cancer in Macedonia was 134.1 out of a population of 100,000, and for 2012, it was 146.4 out of 100,000 (WHO, n.d.).

Women at high risk for breast cancer are defined as one of the following four types: (1) genetic carriers of the more commonly known breast cancer genes (BRCA1, BRCA2, TP53), (2) first-degree relatives of carriers, (3) women with varying degrees of high-risk family histories, (4) and/or women with greater than $20 \%$ lifetime risk for breast cancer based on existing risk models. Genetic carriers for this disease, primarily women with BRCA1 or BRCA2 mutations, 
have a lifetime probability of approximately $85 \%$ of developing breast cancer (Kriege et al., 2004).

Preventive options for these women include surgical interventions such as prophylactic mastectomy (removal of the breasts) and/or oophorectomy (removal of the ovaries). Therefore, it is important to evaluate the benefits and risks of different screening modalities, in an effort to identify additional options for these women. Both of these surgical interventions are practiced at the Bitola clinical hospital.

Screening mammography is effective in women aged 50 years or over, though it is not recommended for average-risk women younger than 50. The Macedonian health system ensures annual sonography for women and mammography every two years after the age of 50. While these methods are significant, it is not the aim of our research to describe their significance.

Macedonia, a middle-economically developed country, has a health system that includes early screening of patients with breast cancer. Therefore, screening modalities other than mammography should be examined, particularly as there may be alternative screening options for certain subgroups of women. Mammography equipment is installed in every regional clinical hospital, and examination is regulated by individual health systems as well as the law. Most patients are female (97.3\%), which aligns with comparative studies that analyze the disease by sex. MRI is more a significant diagnostic method, , but there is a higher cost for this type of examination (Berrington de Gonzalez \& Reeves, 2005; Kriege et al., 2004; MARIBS Study Group, 2006). Mammography, like MRI, has a dose of radiation, and for this reason has special protocols for screening (Law \& Faulkner, 2001; Law, Faulkner, \& Young, 2007; Mattsson $\&$ Rutquvist, 2000). Patients with low risk are screened with sonography, which can be done by doctors of different specialties (Law \& Faulkner, 2002). In addition, some studies state that mammography itself can increase breast cancer due to the dose of radiation (Law \& Faulkner, 2002; National Radiation Protection Board, 2005).

The objective of our study was to examine the frequency of mammography and mastectomies in the Bitola clinical hospital. Students educated at the University St. Clement Ohridski High Medical School, and as a basis for 
practice at the Bitola clinical hospital, have the opportunity to learn from radiological experts and new equipment. Graduated students can and are able to work in their hometowns and be a part of radiological diagnosing teams; for example, they can provide mammography examinations in rural places with portable machines. The target population of their work can be women at high risk for breast cancer who live in rural areas.

\section{References}

Berrington de Gonzalez, A., \& Reeves, G. (2005). Mammographic screening before age 50 years in the UK: Comparison of the radiation risks with the mortality benefits. British Journal of Cancer, 93, 590-596.

Coldman, A., Phillips, N., Warren, L., \& Kan, L. (2006). Breast cancer mortality after screening mammography in British Columbia women. International Journal of Cancer, 120, 1076-1080.

Kriege, M., Brekelmans, C. T. M., Boetes, C., Besnard, P. E., Zonderland, H. M., Objeijn, I. M.... Klijn, J. G. M. (2004). Efficacy of MRI and mammography for breastcancer screening in women with a familial or genetic predisposition. The New England Journal of Medicine, 351(5), 427-437.

Law, J., \& Faulkner, K. (2001). Cancers detected and induced, and associated risk and benefit, in a breast screening program. British Journal of Radiology, 74, 1121-1127.

Law, J., \& Faulkner, K. (2002). Two-view screening and extending the age range: The balance of benefit and risk. British Journal of Radiology, 75, 889-894.

Law, J., Faulkner, K., \& Young, K. C. (2007). Risk factors for induction of breast cancer by $\mathrm{x}$-rays and their implications for breast screening. British Journal of Radiology, 80, 261-266.

MARIBS Study Group. (2006). Screening with magnetic resonance imaging and mammography of a UK population at high familial risk of breast cancer: A prospective multicentre cohort study (MARIBS). Lancet, 365, 1769-1778.

Mattsson, A., \& Rutquvist, L. E. (2000). Radiation risk and mammographic screening of women from 40 to 49 years of age: Effect on breast cancer rates and years of life. British Journal of Cancer, 82(1), 220-226. 
National Cancer Institute of Canada. (2006). Canadian cancer statistics. Toronto: Author. Retrieved from https://www.cancer.ca/ /media/cancer.ca/CW/cancer\%20 information/cancer\%20101/Canadian\%20cancer\%20statistics/Canadian-CancerStatistics-2006-EN.pdf

National Radiation Protection Board. (2005). Recommendations of the International Commission on Radiation Protection. Retrieved from www.icrp-org

World Health Organization (n.d.). Cancer mortality and morbidity. Retrieved April 28, 2015 from http://www.who.int/gho/ncd/mortality_morbidity/cancer/en/

World Health Organization (2014). WHO position paper on mammography screening. Retrieved April 28, 2015 from Retrieved April 28, 2015 from http://apps.who.int/iris/ bitstream /10665/137339/1/9789241507936_eng.pdf?ua=1\&ua=1

Yaffe, M.J. (2004). What should the burden of proof be for acceptance of a new breastcancer screening technique? Lancet, 364(9440), 1111-1112. 